\title{
DOENÇA DE CHAGAS: UMA REVISÃO BIBLIOGRÁFICA
}

CHAGAS DISEASE: A LITERATURE REVIEW

\author{
COSTA, M. ${ }^{1}$; TAVARES, V.R ${ }^{2 ;}$ AQUINO M.V.M. ${ }^{3}$; MOREIRA D.B ${ }^{3}$
}

1. Milce Costa: Docente do curso de Farmácia da FACER- Unidade Ceres. Doutora em Medicina Tropical UFG. milcebiomol@yahoo.com.br. Av. Brasil, S/N, Qd. 13 Morada Verde Ceres - Go. CEP: 76.300-000.

2. Viviane Rodrigues Tavares: Coordenadora / Docente do curso de Enfermagem da FACERUnidade Ceres. Mestre em Enfermagem UFG. vianevivi@ hotmail.com.

3. Marcos Vinícyus Moreira de Aquino, Dhulhya Beibyene Moreira: Acadêmicos do curso de graduação em Farmácia da FACER - Faculdades - Unidade Ceres.

\section{RESUMO}

A doença de Chagas foi descoberta em 1909, por Carlos Ribeiro Justiniano das Chagas. Consiste em uma infecção sistêmica de evolução essencialmente crônica, causada pelo protozoário flagelado Trypanosoma cruzi. A transmissão da doença de Chagas pela via vetorial é considerada o mecanismo de maior relevância epidemiológica. Porém, no Brasil e em vários países da América Latina a principal via de transmissão da infecção chagásica em áreas urbanas é atualmente a via transfusional, ainda considerando outras formas de transmissão. A doença de Chagas encontra-se difundida por todo continente Americano, constituindo um sério problema de saúde pública. Estima-se que o número de pessoas portadoras da infecção chagásica na América Latina varia entre 18-20 milhões de casos, de acordo com a Organização Mundial da Saúde. No Brasil, estima-se que há cerca de três milhões de portadores da doença de Chagas. A prevenção da doença de Chagas engloba vários aspectos a serem considerados como as condições de vida do indivíduo, neste caso deve-se levar em consideração sua habitação, já que moradias inadequadas é domicílio do barbeiro; o inseto vetor; a transmissão via oral; a contaminação congênita; os possíveis acidentes, e a doação de sangue.

Palavras chave: Doença de Chagas; Trypanosoma cruzi; transmissão.

\section{ABSTRACT}

The Chagas disease was discovered in 1907 by Carlos Ribeiro Justiniano das Chagas. Consists of a systemic infection caused by the protozoan Trypanosoma cruzi. The transmission of Chagas disease through vector is considered the most important transmission mechanism. In Brazil and a lot of countries of Latin America the main route of transmission of Chagas infection in urban areas is by transfusion, even considering other ways of transmission. Chagas disease is widespread throughout the American continent, and is a serious public health problem. Is estimated that the number of people with Chagas infection in Latin America is between 18 and 20 million cases, according the World Health Organization. In Brazil is estimated that there are 3 million carriers of Chagas disease. Prevention of Chagas 
disease goes around a lot aspects to consider such as the living condition of the people, in this case, must take in consideration their housing, as inadequate housing is a domiciled barber; the insect vector, oral transmission, congenital contamination; the possible accidents and the blood donation .

Key words: Chagas disease; Trypanosoma cruzi; transmission.

\section{HISTÓRICO}

Em 1909, o médico e cientista brasileiro Carlos Ribeiro Justiniano das Chagas, então pesquisador assistente do Instituto Oswaldo Cruz, mudou-se para a cidade de Lassance, interior de Minas Gerais, com objetivo de combater a malária que acometia os trabalhadores envolvidos na construção dos ramais da estrada de ferro central do Brasil. Neste período, Carlos Chagas utilizou como moradia, laboratório e consultório o vagão de um trem. Por sua genialidade e curiosidade científica, ele examinava animais e pessoas, permanecendo em contato direto com as doenças e a fauna daquela região, de modo que sua dedicação o levou a descobrir um flagelado de mico, o qual denominou Trypanosoma minasense (exclusivo de micos). Após mais estudos, o pesquisador identificou outro tripanossoma, diferente do anterior, de cinetoplasto extremamente denso e movimentação intensa, no tubo digestivo de "chupões" ou "barbeiros", um inseto hematófago muito frequente em casas da região e causador de várias moléstias (FITARELLI; HORN, 2008; NEVES et al, 2005).

Em 1909, Carlos Chagas, diante da descoberta deste último flagelado, enviou amostras de barbeiros infectados ao laboratório de Oswaldo Cruz, no Rio de Janeiro. Ao infectar micos, comprovaram a suspeita de Chagas de que este tripanosoma deveria ser uma espécie nova que circularia entre barbeiros, mamíferos e, talvez, humanos. A partir daí, Carlos Chagas procurou incessantemente aquele protozoário no sangue de pessoas residentes em casas infestadas por barbeiros. A esta nova espécie e em homenagem a Oswaldo Cruz, Chagas a denominou de Trypanosoma cruzi e desta forma descobriu uma nova doença humana, a tripanossomíase americana ou doença de Chagas (CIMERMAN; CIMERMAN, 2008; KROPF; AZEVEDO; FERREIRA, 2000).

Foi assim que no dia 14 de abril de 1909, ao examinar o sangue de uma criança febril, de dois anos de idade, Berenice, que Carlos Chagas identificou o protozoário, antes encontrado em barbeiros. A mãe de Berenice relatou a Chagas que sua filha havia sido sugada por um barbeiro e quais sintomas haviam manifestado (GILBER, 2007; REY, 2008).

A partir de então, Chagas estudou o parasito, seu ciclo evolutivo no vertebrado e invertebrado, insetos vetores e seus reservatórios, a clínica inerente a fase aguda da doença, a patologia, a epidemiologia da doença e a sintomatologia inerente a fase aguda da doença, o seu primeiro diagnóstico parasitológico (gota espessa) e as formas de tratamento. Além disso, tornou notável às autoridades a importância médica e social da doença (GILBER, 2007).

\section{AGENTE ETIOLÓGICO}

A doença de Chagas, uma zoonose, tem como agente etiológico o protozoário monoflagelado Trypanosoma cruzi (SAÚDE-GUIMARÃES; FARIA, 2007). O parasito possui um ciclo biológico complexo do tipo heteroxênico e passa por diferentes formas evolutivas no interior do hospedeiro vertebrado (homem, quatis, mucuras, tatu, morcego, paca, porco-espinho, macacos, gambá, cães, gato, entre outros) e nos insetos vetores: Triatoma infestans, Triatoma sórdida, Triatoma rubrovaria, Triatoma pseudomaculata, Triatoma brasiliensis, Panstrongylus lutzi, Panstrongylus megistus, entre outros. Em todo 
Cone Sul (Argentina, Bolívia, Brasil, Chile, Paraguai e Uruguai) o Triatoma infestans foi o maior responsável pela endemia chagásica (BRASIL, 2009; MELO et al, 2008; REY, 2008; SILVEIRA; DIAS, 2011; SOUSSUMI, 2004).

O ciclo biológico do Trypanosoma cruzi no hospedeiro vertebrado e invertebrado apresenta várias formas evolutivas: tripomastigotas, amastigotas, epimastigotas e esferomastigotas (NEVES et al, 2005) (Figura 1).

As formas tripomastigotas, com comprimento aproximadamente de $20 \mu \mathrm{m}$, são fusiformes e alongadas. Geralmente o núcleo está posicionado no centro e o cinetoplasto (mitocôndria modificada rica em DNA), oval e grande, está situado posteriormente ao núcleo, no entanto, a visualização desta organela só é possível por microscopia eletrônica. Proximamente ao cinetoplasto emerge o flagelo dotado de grande mobilidade. Esta forma ocorre na corrente sanguínea dos hospedeiros vertebrados e na porção distal do tubo digestivo do inseto vetor, não dispondo de capacidade de multiplicação. São estas as formas infectantes para os hospedeiros vertebrados (CIMERMAN; CIMERMAN, 2008; MARKELL et al, 2003) (Figura 2).

As formas amastigotas são esféricas com o cinetoplasto visível, podendo ser encontrada no interior das células de hospedeiros infectados e em cultivo celular. Estas formas se multiplicam por fissão binária e não possuem flagelo (LOZANO, 2011; NASCENTE, 2010) (Figura 3).

As formas epimastigotas e esferomastigotas não ocorrem no hospedeiro vertebrado. Os epimastigotas possuem formas alongadas e seu flagelo surge na porção anterior do parasito com mobilidade muito intensa e se multiplicam por divisão binária simples no vetor ou em meio de cultura. (NEVES et al, 2005) (Figura 2).

Os esferomastigotas por sua vez apresentam a forma esférica do parasito, possuem a capacidade replicativa e são encontrados no estômago do vetor (CIMERMAN; CIMERMAN, 2008). 


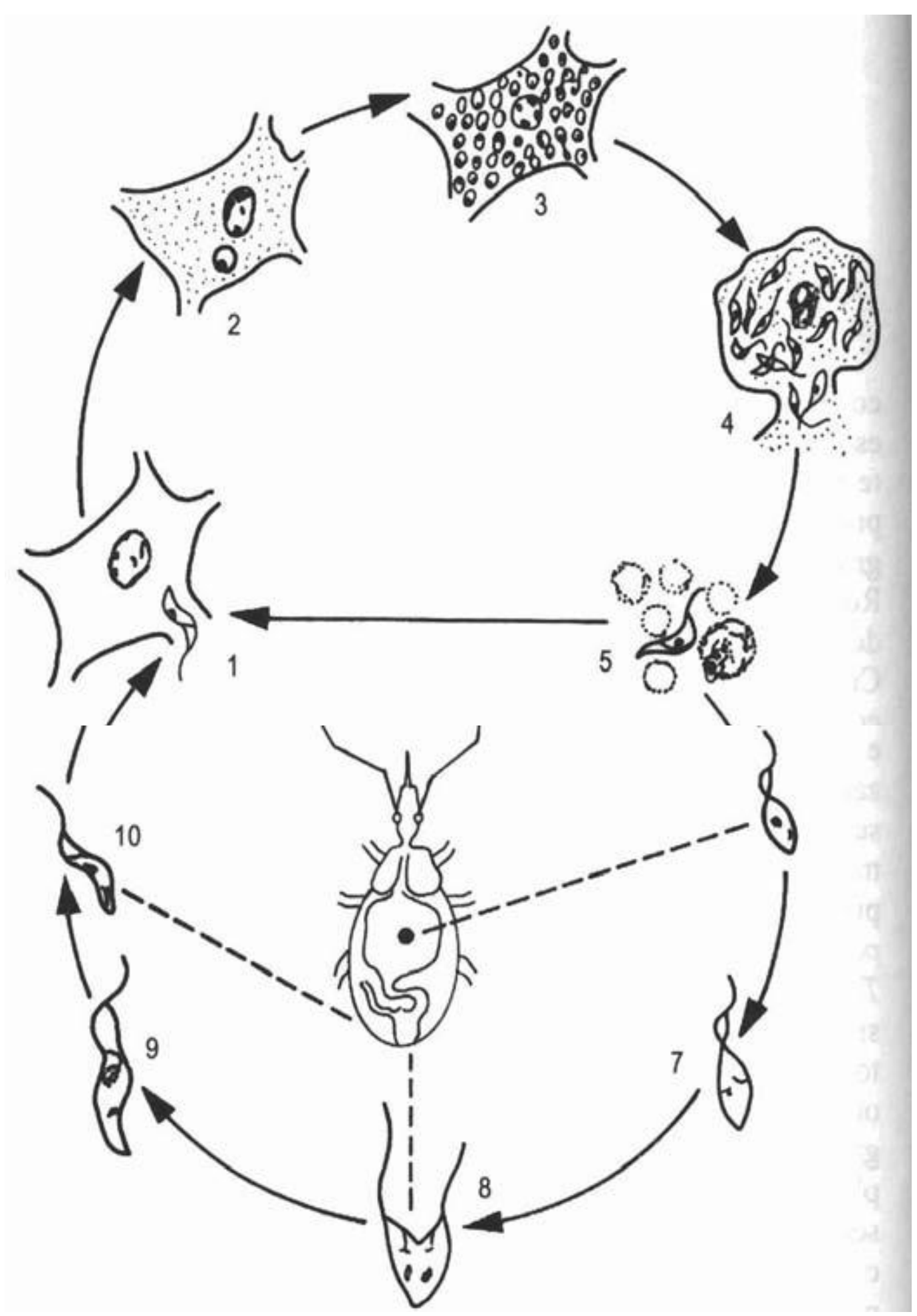

Figura1. Ciclo biológico do T. cruzi. 1) penetração do tripomastigota metacíclica (ou tripomastigota) em uma célula; 2) transformação do tripomastigota em amastigota; 3) essa forma multiplica-se intensamente por divisão binária dentro da célula; 4) rompimento da célula parasitada, liberando tripomastigota; 5) forma tripomastigota no sangue circulante; pode penetrar em outra célula (1) ou ser ingerida pelo triatomíneo $(6)$; 6) forma tripomastigota no estômago do triatomíneo; 7) transformação da forma tripomastigota em epimastigota no intestino posterior do inseto; 8) forma epimastigota em multiplicação por divisão binária; 9) forma epimastigota transforma-se em forma tripomastigota metacíclica no reto do inseto; 10) forma tripomastigota metacíclica, nas fezes do triatomíneo, apta a penetrar em células do hospedeiro mamífero. (Ciclo adaptado de Cançado, R. - Doença de Chagas, 1968.).

Fonte: NEVES et al, 2005. 

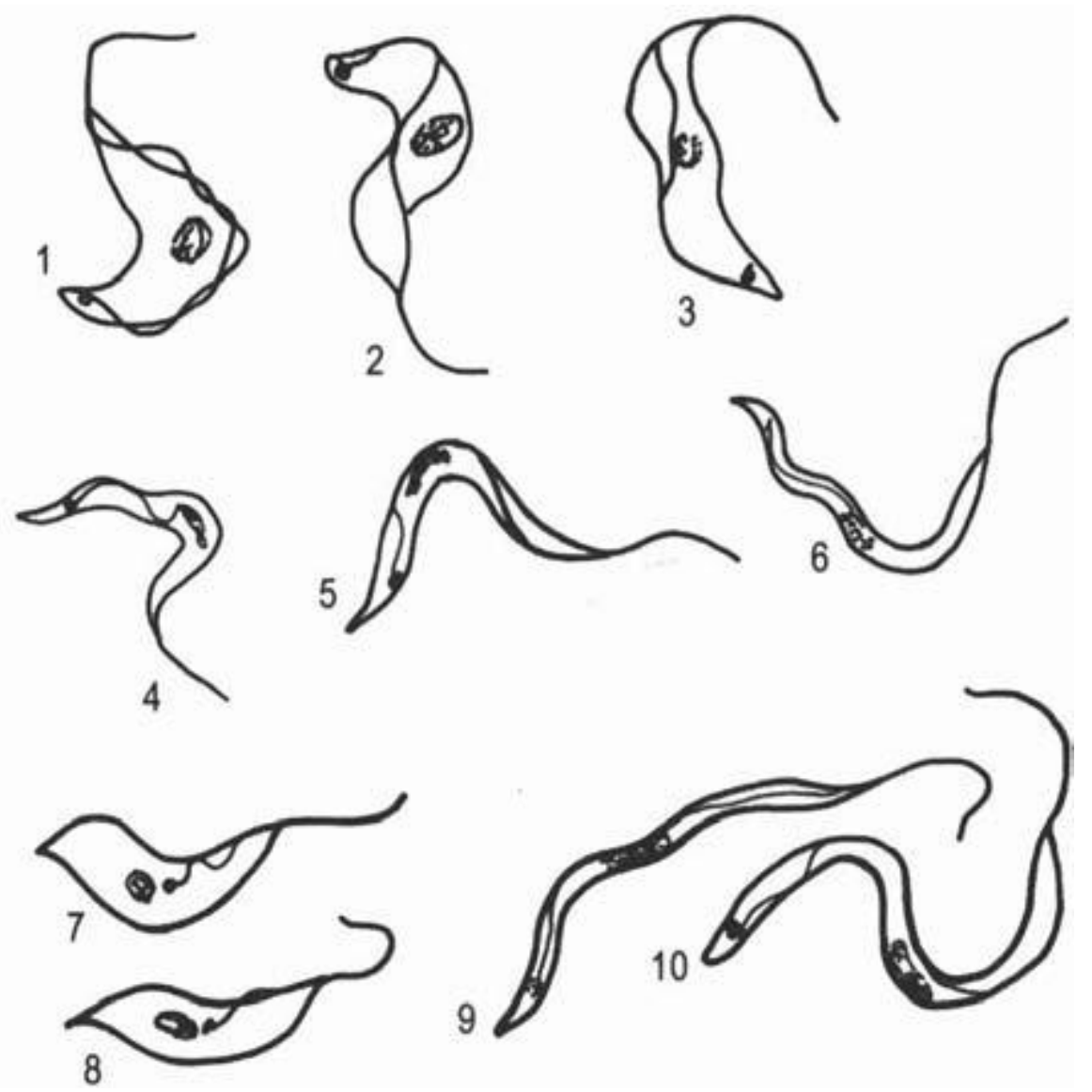

Figura 2. Trypanosoma cruzi: 1, 2 e 3, formas tripomastigotas largas, encontradas no sangue circulante; 4, 5 e 6 , formas tripomastigotas delgadas, encontradas no sangue circulante; 7 e 8 , formas epimastigotas encontras em dejetos de triatomíneos e meios de cultura; 9 e 10, formas tripomastigotas encontradas em dejetos de triatomíneos e meios de cultura. Fonte: NEVES et al, 2005.

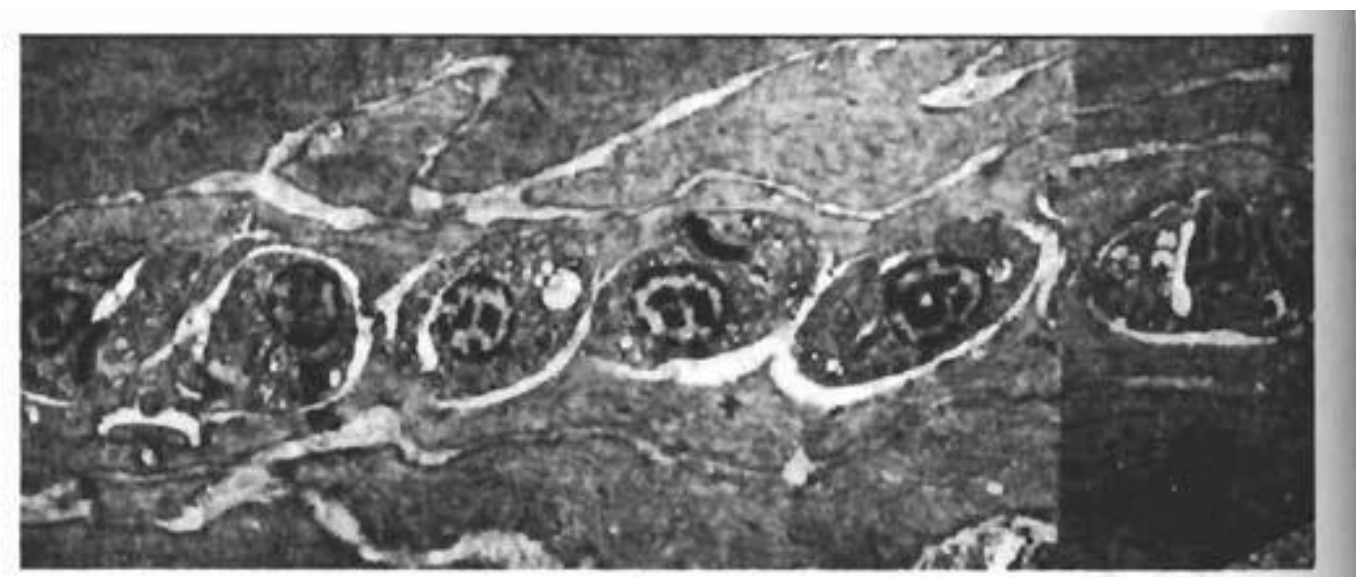

Figura 3. Trypanosoma cruzi: formas amastígotas no tecido muscular.

Fonte: NEVES et al, 2005. 


\section{TRANSMISSÃO DA DOENÇA DE CHAGAS}

A transmissão da doença de Chagas pela via vetorial é considerada o mecanismo de transmissão de maior relevância epidemiológica, correspondendo a $80 \%$ dos casos da doença (ARAS et al, 2003). No entanto, estudos recentes mostram que no Brasil e em vários países da América Latina a principal via de transmissão da infecção chagásica em áreas urbanas atualmente é a via transfusional (SAÚDE-GUIMARÃES; FARIA, 2007; SOBREIRA et al, 2001). Entretanto, não se devem desconsiderar os demais meios de transmissão tais como a via congênita e as consideradas vias de exceção, as quais não representam valor significativo na saúde pública, como a via oral, acidental e transplantar, entre outras (MORAES-SOUZA; FERREIRA-SILVA, 2011; SAÚDE-GUIMARÃES; FARIA, 2007).

\subsection{TRANSMISSÃO VETORIAL}

No âmbito da via de transmissão vetorial é necessário que ocorra a interação vetorhospedeiro, sendo o vetor o principal elo na cadeia dessa modalidade de transmissão (SILVEIRA; DIAS, 2011). O T. cruzi desenvolve-se com sucesso nos insetos da família Reduviidae e subfamília Triatominae. Os triatomíneos, como são conhecidos os insetos vetores, são considerados os vetores mais importantes da doença. O parasito é transmitido a mamíferos, incluindo o homem, pelo inseto vetor, que popularmente é conhecido como "barbeiro" ou "chupão". Este inseto possui hábitos noturnos e apresenta em sua saliva propriedade anestésica e anticoagulante, tornando assim indolor sua picada. Durante ou logo após o hematofagismo o vetor defeca. A importância do momento da evacuação está no fato dos tripanossomos desenvolverem-se no intestino posterior do triatomíneo e serem disseminados nos dejetos do inseto (TARTAROTTI et al, 2004). Normalmente, ocorre uma leve ardência ou coceira no local da picada, assim, quando a pessoa se coça, acaba por propiciar a penetração do parasito contido nas excreções do barbeiro no organismo, causando a infecção (ARGOLO et al, 2007).

Uma vez contaminado com o parasito, o triatomíneo continua infectado por toda sua existência, variando seu potencial de infecção de acordo com a cepa do parasito e peculiarmente com a sua capacidade em aderir-se ao espaço domiciliar (CIMERMAM; CIMERMAM, 2008).

No Brasil a transmissão vetorial foi substancialmente reduzida frente aos avanços tecnológicos utilizados no controle das atividades de vigilância epidemiológica, juntamente com estratégias para a identificação da presença do vetor, desencadeando assim ações de combate ao mesmo. O controle da transmissão vetorial foi realizado com êxito em diversos países do Cone sul (FERREIRA; SILVA, 2006; SOBREIRA et al, 2001).

\subsection{TRANSMISSÃO CONGÊNITA}

Outro meio de transmissão da doença de Chagas consideravelmente relevante é a transmissão congênita (ORGANIZACIÓN PANAMERICANA DE LA SALUD, 2007). A transmissão vertical da doença de Chagas ocorre principalmente pela via transplacentária, que pode ocorrer tanto na fase aguda quanto na fase crônica da doença, podendo ocorrer em qualquer período da gestação, sendo mais provável no último trimestre. Porém, a transmissão também pode ocorrer no canal do parto, através do contato de mucosas do feto com o sangue da mãe contaminado por T. cruzi (GONTIJO et al, 2009).

Havendo a contaminação, as crianças, normalmente apresentam baixo peso ao nascer. Nestes casos, o número de natimortos é consideravelmente alto (MARKELL et al, 2003). Sendo considerada inevitável, cabe então uma possível prevenção secundária, que é diagnosticar precocemente a infecção nos recém-nascidos filhos de mães chagásicas. Conhecendo o risco de transmissão em determinadas áreas, recomenda-se introduzir no pré- 
natal sorologia própria ao diagnóstico da doença de Chagas. Dessa forma, pode-se iniciar rapidamente o tratamento dos nascidos comprovadamente infetados (SILVEIRA, 2011).

\subsection{TRANSMISSÃO ORAL}

Nos últimos anos casos por transmissão oral tem sido relatado em diversos estados brasileiros. Por via oral a contaminação ocorre pela ingestão de alimentos contendo tripanossomos provenientes de triatomíneos ou suas dejeções (CAVALCANTI et al, 2009). No estado de Santa Catarina, em 2005 houve a suspeita de 45 casos de doença de Chagas aguda ligados a ingestão de caldo de cana. Dentre estes casos, foram confirmados laboratorialmente 31, sendo registrados óbitos de 5 pessoas (MELO et al, 2008). A amamentação também pode ser ressaltada como outro meio de contaminação por via oral. Estudos comprovaram achados de Trypanosoma cruzi no leite de mães com infecção chagásica nas fases aguda e crônica da doença (LAMOUNIER; MOULIN; XAVIER, 2004).

\subsection{TRANSMISSÃO ACIDENTAL}

Acidentalmente a transmissão pode ocorrer em diversas circunstâncias e na sua maioria são despercebidos ou não diagnosticados. Um conjunto de elementos como desconhecimento, desatenção, falta ou mal uso de equipamentos de proteção individual, instalações e equipamentos inadequados e falta de capacitação, são considerados fatores de risco para a contaminação pelo $T$. cruzi . Em laboratórios com indivíduos que manipulam o parasito no sangue de animais, fezes de triatomíneos, pessoas contaminadas, ou vetores infectados, a infecção pode ocorrer pelo contato do parasito com alguma lesão na pele, mucosa oral ou auto-inoculação. Diante destas situações, faz-se importante ressaltar a necessidade das medidas de biossegurança (AMATO NETO, 2011; CIMERMAN; CIMERMAM, 2008; DIAS; NEVES et al, 2005).

\subsection{TRANSMISSÃO TRANSPLANTAR}

O transplante de órgãos também é uma maneira de transmissão citada na literatura científica. Os primeiros relatos de contaminação por esse meio de transmissão datam do início de 1980 através de um transplante de rim. No que se refere à via transplantar, essa só ocorre quando não há triagem no processo de transplantes. Ocorrendo a transmissão, o indivíduo pode desenvolver a fase aguda grave, já que o indivíduo transplantado, contaminado, apresenta menor resistência à infecção, devido ao tratamento com imunossupressores (DIAS; AMATO NETO, 2011).

\subsection{TRANSMISSÃO TRANSFUSIONAL}

A transmissão transfusional da doença de Chagas foi confirmada pela primeira vez no Brasil em 1952 por Pedreira de Freitas, em São Paulo, relatando dois casos de pacientes infectados por esta via (SILVA, 2010).

O processo migratório no sentido rural-urbano nas últimas décadas em função da industrialização do Brasil promoveu o fenômeno de urbanização dos "chagásicos". Esse fato foi responsável pela alta prevalência de doadores chagásicos nos bancos de sangue do país (MORAES-SOUZA; FERREIRA-SILVA, 2011). Estima-se que 60\% dos indivíduos contaminados residem em espaços urbanos e nas grandes metrópoles e em $50 \%$ destes o parasito é identificado na fase crônica constituindo um grupo de potenciais doadores de sangue, aumentando, assim, o risco da doença de Chagas transfusional (CIMERMAN; CIMERMAM, 2008). 
Na década de 70 as doações de sangue se concentravam nos grandes centros urbanos e o aparato tecnológico para coleta de sangue era rudimentar. Nessa época os doadores eram remunerados e em grande número e não havia controle do sangue transfundido. Em 1976, o Ministério da Saúde definiu a necessidade de se implantar hemocentros públicos, baseados na doação voluntária de sangue (SILVA, 2010).

Foi somente na VIII Conferência Nacional de Saúde ocorrida em 1986, que se discutiu a questão da transmissão da doença de Chagas através da transfusão sanguínea. Em 1988, a nova Constituição brasileira ampliava a cobertura química contra o vetor para cerca de 2.450 municípios. Além disso, proibiu o doador remunerado, normatizou a prática da hemoterapia, criou um sistema nacional de sangue e hemoderivados e instituiu a obrigatoriedade da seleção sorológica dos candidatos à doação. Dessa forma, essas medidas contribuíram para a diminuição da prevalência da doença em doadores de sangue (DIAS, 2006).

Mesmo diante destas ações, vários são os fatores que propiciam a persistência do risco de transmissão transfusional da doença de Chagas. Os principais fatores envolvem: falhas na triagem clínica e sorológica, a prevalência da doença na região, a forma e quantidade de produto sanguíneo infectado transfundido, a situação imune do receptor, o baixo nível de cobertura da sorologia para $T$. cruzi nos serviços de hemoterapia e o grau de sensibilidade dos testes para diagnóstico sorológicos utilizados nos possíveis doadores (FERREIRA-SILVA, 2011; MORAES-SOUZA; SILVA, 2010).

\section{MANIFESTAÇÕES CLÍNICAS}

A doença de Chagas é um problema preocupante pela enorme quantidade e variedades de danos que envolvem o paciente chagásico tornando-se danosa em suas consequências (GILBER, 2007).

Clinicamente a doença de Chagas apresenta duas fases bastante distintas: fase aguda e fase crônica. A fase aguda apresenta-se sintomática ou assintomática, sendo mais frequente na primeira infância (LOZANO, 2011). Essa fase inicia-se quando o parasito penetra no vertebrado. A manifestação sintomática inicia de oito a dez dia após a entrada do agente etiológico na corrente sanguínea do hospedeiro vertebrado. Nesta fase, o indivíduo pode apresentar sinais de porta de entrada da infecção, como sinal de Romaña e o Chagoma de inoculação. No sinal de Romaña ocorre um edema indolor na pálpebra inferior e superior de um dos olhos, simultaneamente ocorre uma coloração palpebral eritematoso-violácea, congestão conjuntival e linfonodomegalia satélite. O Chagoma de inoculação consiste em um pequeno nódulo eritematoso que pode surgir em qualquer região do corpo, no entanto, é mais frequente nas regiões descobertas durante o sono. As manifestações gerais são: febre, astenia, inapetência e cefaléia. Podem surgir outros sintomas como linfonodomegalia generalizada e hepatoesplenomegalia, e até manifestações neurológicas características de meningo-encefalite e manifestações cardíacas. Os sintomas da fase aguda normalmente desaparecerem de quatro a oito semanas na maioria dos indivíduos acometidos (COLOSIO, 2007; SANTOS, 2011).

Após o término da fase aguda inicia-se a fase crônica. Esta fase é subdividida em crônica assintomática (forma indeterminada ou latente) e crônica sintomática. A forma indeterminada ou latente caracteriza-se por achados clínicos e laboratoriais como: inexistência de manifestações clínicas (sinais e/ou sintomas da doença) significantes; eletrocardiograma sem significativas alterações ou coração, esôfago e cólon radiologicamente normal e sorologia reagente, normalmente, diagnosticado em bancos de sangue. Aliás, a forma indeterminada ou latente é a mais frequente forma clínica da tripanossomíase identificada na população das áreas endêmicas e entre doadores de sangue. Trinta por cento dos pacientes chagásicos que apresentam a forma indeterminada irão manifestar alguma das formas crônicas da tripanossomíase após um período de 10 a 20 anos (BARBOSA, 2009; MEYER, KANESHIMA, SOUZA-KANESHIMA, 2006). 
$\mathrm{Na}$ fase crônica sintomática, certa parcela dos chagásicos, após permanecerem assintomáticos por vários anos, podem apresentar com o tempo, complicações relacionadas ao sistema cardiovascular e digestivo. Isso se deve ao fato de mudanças anatômicas ocorridas no miocárdio e no tubo digestivo. Nesta fase, observa-se reativação intensa do processo inflamatório (GILBER, 2007).

A forma crônica cardíaca da doença é a mais relevante pela elevada causa de morbimortalidade pelos diversos danos ocorridos no sistema de condução e pela falência cardíaca nos casos graves da Doença de Chagas (LUNARDELLI et al, 2007). Há evidências que o acometimento cardíaco evolui para quadros de miocardiopatia dilatada e insuficiência cardíaca congestiva. A miocardite é silenciosa e leva a perda progressiva da massa miocárdica, promovendo certa destruição e como consequência provoca dilatação cardíaca e/ou disritmia potencialmente fatal. Na miocardiopatia encontra-se infiltrado mononuclear com regiões de adensamento celular, fibrose, dissociação, fragmentação e hialinização de fibras cardíacas (ALBAJAR et al, 2003). Essa forma ocorre em aproximadamente $30 \%$ dos casos crônicos, sendo a principal responsável pela mortalidade dos pacientes chagásicos (BRASIL, 2009; SOUSSUMI, 2004).

No Brasil, a forma digestiva ocorre em cerca de $10 \%$ dos casos, variando dependendo da região geográfica estudada. As manifestações digestivas são caracterizadas por lesões dos plexos intramurais em virtude do parasitismo das células musculares vizinhas, refletindo na função motora, especialmente do esôfago e do cólon, fazendo com que a musculatura lisa desses órgãos responda com contrações desordenadas. No cólon de pacientes chagásicos pode haver dilatações dos cólons (sigmóide e reto), caracterizando megacolón (SANCHEZLERMEN et al, 2007; SILVA, 2011).

Quando transmitida por transfusão, a doença de Chagas apresenta-se oligo ou assintomaticamente na maioria dos casos manifestando a fase aguda de modo excepcional em pacientes imunossuprimidos como portadores de AIDS, transplantados e pacientes em tratamento com quimioterápicos. Similar à transmissão vetorial, em indivíduos imunocompetentes a infecção chagásica adquirida pela via transfusional raramente é desenvolvida e o período de incubação costuma ser mais extenso, podendo ultrapassar os 100 dias. Quando ocorrem manifestações clínicas, essas são caracterizadas por febre prolongada, linfadenopatia e hepatoesplenomegalia (MORAES-SOUZA; FERREIRA-SILVA, 2011).

\section{INCIDÊNCIA E PREVALÊNCIA}

O número de pessoas portadoras da infecção chagásica na América Latina varia entre 18-20 milhões de casos, de acordo com a Organização Mundial da Saúde (OMS). No Brasil, estima-se que há cerca de três milhões de portadores da doença de Chagas. Sendo Goiás o estado brasileiro que representa maior taxa da doença (SILVA et al, 2010; SOBREIRA et al, 2001).

Antigamente, precisamente nos anos 70, a área correspondente a endemia por doença de Chagas, incluía 18 estados com mais de 2.200 municípios, dentre os quais se constatou a presença do Triatoma infestans em 711 destes municípios (BRASIL, 2012). Porém com a iniciativa do Cone Sul, sob a coordenação da Organização Pan-americana da Saúde e com o apoio dos governos dos países envolvidos, conseguiu-se programar a integração de campanhas de saúde pública, investimentos financeiros e ações de controle da endemia, obtendo resultados satisfatórios no aspecto epidemiológico da transmissão vetorial (ARAS et al, 2003). Com isso, no ano de 2006 o Brasil obteve pela Organização Pan-Americana de Saúde e pela OMS certificado da interrupção da transmissão vetorial da infecção chagásica. É importante ressaltar que essa certificação não significa o controle efetivo da doença no Brasil, mas representa em caráter momentâneo, a exclusão da transmissão típica pelo triatomíneo da espécie Triatoma infestans (FERREIRA; SILVA, 2006). 
A Amazônia é hoje a área que apresenta maior número de casos da doença de Chagas. No período de 1997 a 2008 foram registrados, 617 casos da doença de Chagas aguda, considerados como pequenos surtos. Na maioria das vezes esses surtos estão intimamente ligados à transmissão oral, devido à ingestão de sucos de frutas regionais, como o açaí (BARBOSA-FERREIRA et al, 2010). Sendo assim, hoje, a região Amazônia é contemplada como área endêmica para doença de Chagas (PINTO et al, 2008).

Segundo dados do Ministério da Saúde, no período de 2000 a 2011, houve o registro de 1.252 casos da doença de Chagas no Brasil. Setenta por cento destes casos aconteceram por transmissão oral, em $7 \%$ a transmissão ocorreu por via vetorial e em $22 \%$ dos casos não se identificou a forma de transmissão (BRASIL, 2012).

A Tabela 1 demonstra o número de casos de doença de Chagas aguda, registrados no período de 2007 a 2011 em todo o Brasil, dividindo por regiões e estados, e a quantidade de casos de cada ano em determinada região e estado (BRASIL, 2012).

Tabela 1: Casos de doença de Chagas Aguda. Brasil, Grandes Regiões e Unidades Federadas, 2007 a 2011.

\begin{tabular}{|c|c|c|c|c|c|c|}
\hline Regiăo/ UF & 2007 & 2008 & 2009 & 2010 & 2011 & TOTAL \\
\hline Norte & 157 & 124 & 248 & 91 & 148 & 768 \\
\hline RO & 0 & 0 & 0 & 1 & 1 & 2 \\
\hline AC & 0 & o & 2 & 5 & 0 & 7 \\
\hline AM & 28 & 0 & 3 & 23 & 12 & 66 \\
\hline RR & 0 & 0 & o & o & 0 & 0 \\
\hline PA & 109 & 99 & 233 & 59 & 113 & 611 \\
\hline AP & 19 & 20 & 10 & 3 & 7 & 64 \\
\hline то & 1 & 5 & 0 & o & 15 & 21 \\
\hline Nordeste & 3 & 7 & 1 & 21 & 10 & 42 \\
\hline MA & 2 & 5 & 1 & 0 & 9 & 17 \\
\hline PI & 1 & 1 & o & 8 & 1 & 11 \\
\hline CE & o & 1 & o & o & 0 & 1 \\
\hline R N & o & o & o & o & 0 & o \\
\hline PB & 0 & 0 & o & 2 & 0 & 2 \\
\hline PE & o & o & o & 9 & 0 & 9 \\
\hline AL & 0 & 0 & o & o & 0 & o \\
\hline SE & 0 & 0 & o & 2 & 0 & 2 \\
\hline BA & o & 0 & o & o & 0 & o \\
\hline Sudeste & o & o & o & o & 1 & 1 \\
\hline MG & 0 & 0 & 0 & o & 0 & o \\
\hline ES & 0 & o & o & o & 0 & o \\
\hline RU & 0 & o & o & o & 1 & 1 \\
\hline SP & 0 & 0 & o & o & 0 & o \\
\hline Sul & o & o & 4 & 3 & o & 7 \\
\hline PR & o & 0 & 4 & 2 & o & 6 \\
\hline sc & 0 & 0 & o & 0 & 0 & o \\
\hline RS & 0 & 0 & o & 1 & 0 & 1 \\
\hline Centro-Oeste & 1 & o & 5 & 18 & 7 & 31 \\
\hline MS & o & 0 & 5 & 3 & o & 8 \\
\hline мT & 1 & o & o & o & 1 & 2 \\
\hline Go & 0 & 0 & o & 15 & 6 & 21 \\
\hline DF & o & o & 0 & o & 0 & o \\
\hline Brasil & 161 & 131 & 258 & 133 & 166 & 849 \\
\hline
\end{tabular}

Disponível em < http://portal.saude.gov.br/portal/arquivo/pdf/gve_7ed_web_atual.pdf>

Mediante a realização de combate ao vetor, a doença de Chagas transfusional se tornou o principal meio de transmissão na maior parte dos países endêmicos. Contudo, a prevalência da doença em doadores de sangue no Brasil era de $8,3 \%$ nos anos 50, caindo para $6,9 \%$ nos anos 60 e 70 e, 3,2\% no fim dos anos 80 e início de 90. Em 2006 o documento da Organização Mundial da Saúde apresentou um índice de prevalência de doadores de sangue 
contaminados por $T$. cruzi de 1,3\% em toda América Latina e de apenas 0,2\% no Brasil (MORAES-SOUZA; FERREIRA-SILVA, 2011).

Estima-se que esse baixo índice de prevalência da doença de Chagas seja proveniente das medidas de melhoria da qualidade dos serviços de hemoterapia realizadas a partir da década de 80 na maioria dos estados brasileiros e ao novo caráter de doação, agora voluntária (MELO et al, 2008).

\section{DIAGNÓSTICO CLÍNICO}

Em virtude da sintomatologia característica e/ou alterações em exames laboratoriais, alguns pacientes buscam atendimento médico e por vezes são diagnosticados com a doença de Chagas. Algumas causas proporcionam a ida do paciente ao médico como: exame sorológico positivo para Chagas, eletrocardiograma anormal, falta de ar ao se esforçar, palpitações, perda de consciência ou outras manifestações de insuficiência cardíaca, disfagia ou obstipação prolongada (REY, 2008).

Em regiões endêmicas, no entanto, o fato de crianças apresentarem febre, com poliadenite, espatoesplenomegalia e sintomas cardíacos deve-se cogitar a possibilidade de tripanossomíase. Durante a fase aguda, o diagnóstico clínico é facilitado com a presença de sinais de porta de entrada do parasito como sinal de Romaña e/ou Chagoma de inoculação (REY, 2008).

Existem ainda alguns dados relacionados aos pacientes que são importantes ao se investigar suspeitas clínicas da doença de Chagas como o histórico de antecedentes de contato com material contendo o parasito ou os vetores; a receptividade de transfusões sanguíneas ou transplante de órgão recente; a ingestão de produtos alimentícios artesanais e sem cozimento e a ingestão de alimentos expostos aos vetores ou reservatórios (ORGANIZAÇÃO PANAMERICANA DA SAÚDE, 2009).

\section{DIAGNÓSTICO LABORATORIAL}

Diante de uma suspeita clínica de tripanossomíase é necessário recorrer à confirmação diagnóstica da doença. Dessa forma, os métodos laboratoriais são de extrema importância para um diagnóstico adequado. No entanto, os métodos de diagnóstico laboratorial podem apresentar diferentes resultados dependendo da fase da doença em que são aplicados, ou seja, fase aguda ou crônica (NASCENTE, 2010).

Durante a fase aguda, ocorre alta parasitemia (presença da forma tripomastigota metacíclica no sangue) e presença de imunoglobulinas específicas anti- $T$. cruzi da classe IgM. Neste período, recomenda-se exame parasitológico de sangue: lâmina corada de gota espessa ou esfregaço, método enriquecido por concentração e a pesquisa a fresco de tripanossomatídeos e exames sorológicos: hemaglutinação indireta ou passiva (HAI), imunofluorescência indireta (IFI) e o método imunoenzimático (ELISA) (BRASIL, 2009).

$\mathrm{Na}$ fase crônica, observa-se baixa parasitemia e altos títulos de imunoglobulinas específicas. Nesta fase, os exames sorológicos mais indicados são IFI, ELISA, HAI e fixação do complemento (não utilizada mais pelos laboratórios da rede pública de saúde). Também podem ser úteis métodos de exames parasitológicos indiretos, como xenodiagnóstico (não utilizado na rotina laboratorial) e hemocultura (ALMEIDA; SANTILIANO, 2012).

Os testes sorológicos, largamente utilizados na rotina laboratorial, apresentam uma grande sensibilidade, são facilmente executados e fornecem resultados em curto prazo (minutos ou horas), sendo de suma importância na triagem de doadores de sangue. O diagnóstico sorológico para doença de Chagas confirma a presença de anticorpos específicos no soro do paciente muito cedo (fase aguda da infecção) e se mantém contínuo durante toda a fase crônica da doença. Apesar disso, não se pode excluir a probabilidade de falsos positivos e falsos negativos, motivo pelo qual se preconiza o emprego de dois métodos diferentes. A 
HAI, a IFI, a aglutinação direta com 2-mercaptoetanol (2-ME) e o ELISA são os métodos recomendados pela OMS e mais utilizados na rotina laboratorial. Na prática clínica, a triagem de doadores de sangue utiliza testes sorológicos de HAI, IFI e ELISA (LUNARDELLI et al, 2007). No entanto, a ANVISA na RDC 57 DE 2010 determina a obrigatoriedade de apenas uma única técnica de alta sensibilidade para a detecção de anticorpo anti-T. cruzi na triagem de doadores de sangue (ANVISA, 2010).

\section{HEMAGLUTINAÇÃO INDIRETA OU PASSIVA (HAI)}

É uma metodologia muito simples que possui sensibilidade maior que 90\%, utilizada para o diagnóstico de fase aguda e crônica. A preparação do reagente consiste em fixar hemácias aos componentes protéicos (antígeno) do T. cruzi com o soro do paciente. Na presença de anticorpos específicos ocorre o processo de aglutinação da preparação (NEVES et al, 2005).

Em bancos de sangue e em inquéritos epidemiológicos foi desenvolvido um teste de hemaglutinação rápido que utiliza hemácias de carneiro sensibilizadas com extrato de $T$. cruzi, em suspensão concentrada. A preparação é feita sobre uma lâmina de vidro, duas gotas de soro ou plasma e uma de reagente, em seguida, agitado em movimentos rotativos e a leitura é feita após 3 minutos (REY, 2008).

\subsection{IMUNOFLUORESCÊNCIA INDIRETA (IFI)}

Caracteriza-se por ser um método com grande sensibilidade na fase aguda. É a mais utilizada atualmente. Consistem em fazer reagir antígenos fixos em lâminas de microscópio, anticorpos do soro do paciente e posteriormente o conjugado (antiimunoglobulina marcada com substância fluorescente). $\mathrm{O}$ episódio de fluorescência em microscópio de fluorescência revela a presença de anticorpo (NEVES et al, 2005).

A imunofluorescência pode ser utilizada para detectar $\operatorname{IgM}$ antitripanossomo, típica da fase aguda da doença, desde que, o conjugado seja específico, anti-IgM. No entanto, é preferível a utilização de IgG que não oferecem riscos de reações cruzadas e reações positivos falsos (REY, 2008).

\subsection{TESTE IMUNOENZIMÁTICO (ELISA)}

Conhecido pela sigla ELISA (do inglês Enzyme Linked Immuno Sorbent Assay), este método oferece vantagens sob as demais técnicas sorológicas por poder ser automatizado e realizar uma grande quantidade de testes de uma só vez. O princípio básico deste método consiste na imobilização de um dos regentes (antígeno ou anticorpo) em fase sólida, em seguida adiciona a amostra, posterior a amostra, adiciona outro reagente ligado a uma enzima (conjugado) que por sua vez reagirá com o complexo antígeno-anticorpo. A fixação do conjugado imunoenzimático é revelada ao adicionar um substrato da enzima e um cromógeno formará um produto colorido que poderá ser medido por espectrofotometria (CAVALCANTI; LORENA; GOMES, 2008).

\section{TRATAMENTO}

A terapêutica da doença de Chagas prossegue parcialmente ineficaz, apesar do real progresso alcançado nas últimas décadas. Diversas drogas vêm sendo estudadas, porém, nenhuma consegue eliminar a infecção pelo $T$. cruzi e promover a cura definitiva da tripanossomíase, permitem apenas efeitos supressivos. Algumas drogas foram ensaiadas na terapêutica da doença de Chagas, mas até agora, somente dois compostos ativos se revelaram 
favoráveis, sendo eles o nifurtimox (Lampit, também conhecido como Bayer 2502) e benzonidazol (Rochagan) (SAÚDE-GUIMARÃES; FARIA, 2007; SILVA, 2010).

$\mathrm{O}$ tratamento antitripanosomal da doença de Chagas com os fármacos benzonidazol e nifurtimox são recomendados para todos os casos agudos. Estes atingem um índice de cura superior a $80 \%$, quando administrados nesta fase. $\mathrm{Na}$ fase crônica estes fármacos apresentam apenas $8 \%$ a $30 \%$ de taxa de cura (OLIVEIRA et al, 2008).

O mecanismo de ação do nifurtimox, 3-metil-4 (5'-nitrofurfurilidenamino)- tetraidro (1,4)-tiazina-1-1-dióxido, consiste na inibição do desenvolvimento intracelular do parasito. Atua bem contra as formas sanguíneas e parcialmente contra as formas teciduais. É administrado oralmente, sob forma de comprimido, por três meses, sendo mais bem tolerado por pacientes jovens (CIMERMAN; CIMERMAN, 2008; MARKELL et al, 2003; NEVES et al, 2005; REY, 2008).

O benzonidazol por sua vez é uma droga nitroheterocíclica (N-benzil-2nitroimidazole-1-acetamida) introduzido na terapêutica da doença de Chagas em 1967. É considerado mais efetivo que o nifurtimox, porém, apresenta moderada toxicidade. $\mathrm{O}$ benzonidazol possui uma consistente capacidade antiprotozoária conseguindo eliminar os parasitas sob as formas sanguíneas e teciduais, se administrado na dose certa e durante o período de dois meses na fase aguda da doença (BEZERRA, MENEGUETTI; CAMARGO, 2012; OLIVEIRA et al, 2008).

No Brasil, o benzonidazol é a única droga atualmente disponível para o tratamento da tripanossomíase americana (PONTES et al, 2010). O nifurtimox, existente na América Central, pode ser utilizado como alternativa em casos não satisfatórios como a intolerância ao benzonidazol (BRASIL, 2005).

O tratamento em crianças ou nos acometidos com a forma crônica indeterminada ou formas cardíacas ou digestivas brandas é recomendado com o objetivo de diminuir o curso evolutivo da doença, diminuir a possibilidade de transmissão do parasito e principalmente a cura da infecção (BRASIL, 2009).

\section{MEDIDAS PREVENTIVAS}

As ações de impedimento da transmissão do parasito ao indivíduo suscetível caracterizam-se como prevenção primária sendo o meio mais efetivo no que se refere à prevenção (CIMERMAM; CIMERMAM, 2008). Porém a prevenção da doença de Chagas engloba vários aspectos a serem considerados como as condições de vida do indivíduo, neste caso deve-se levar em consideração sua habitação, já que moradias inadequadas é domicílio do barbeiro; o inseto vetor; a transmissão via oral; a contaminação congênita; os possíveis acidentes, e a doação de sangue (NEVES et al, 2005).

Considerando que o Triatoma infestans se aloja em casas e nos ambientes peridomiciliares, a melhoria da habitação é retratada como o modo mais eficaz e importante na prevenção, já que, sem condições que propiciam a colonização do triatomíneo no ambiente domiciliar a interação vetor-homem se torna mais difícil (BEDIN et al, 2001). Contudo, a melhoria da habitação, como uma medida de prevenção essencial, deve ser reforçada por meio de ações de caráter educativo envolvendo a sociedade (BRASIL, 2003).

Dessa forma, a prevenção da transmissão vetorial da doença consiste basicamente em eliminar ou reduzir o contato do homem com o vetor, sendo a retirada de triatomíneos no âmbito domiciliar um meio lógico de prevenção (SILVEIRA; DIAS, 2011). Portanto, o uso de inseticidas ainda é o principal meio de controle ao barbeiro. O inseticida organoclorado BHC (Hexaclorociclohexano) já foi o mais utilizado, porém, devido ser altamente tóxico seu uso foi substituído por inseticidas piretróides, já que são menos tóxicos e sua ação residual persiste por mais tempo (ARGOLO et al, 2007).

Quanto à prevenção da transmissão por via oral, seu caráter aleatório e esporádico torna esta situação relativamente difícil de controlar. Medidas de higiene e seleção de 
alimentos adequada se tornaram imprescindíveis em áreas com presença de triatomíneos. Em caso de lactentes de mães contaminadas é aconselhável a suspensão da amamentação caso essas estejam em fase aguda da doença ou em quadro de esquizotripanose, ou em estágio crônico que apresentem rachaduras ou sangramento nos mamilos e aréolas (DIAS; AMATO NETO; LUNA, 2011).

A respeito da contaminação por meio da transfusão sanguínea, o controle do doador de sangue é indispensável. São estabelecidos alguns critérios para a profilaxia neste tipo de contaminação, tais como: a) a seleção dos doadores assegurando cobertura integral na triagem pré-estabelecida para a doação, onde são realizados testes sorológicos com no mínimo duas técnicas de alta sensibilidade, sendo que este critério é previsto por lei no Brasil; b) a quimioprofilaxia realizada pela adição de agente tripanocida - violeta-de-genciana - a 1:4.000 em sangues suspeitos, visto que só exerce esse efeito após um período de 24 horas da aplicação. A violeta é a única droga capaz de exercer função quimioprofilática; c) o uso de filtros celulares para leucócitos inclui outra possibilidade, já que possuem a capacidade de reter o parasito, porém são de alto custo (CIMERMAN; CIMERMAM, 2008; SAÚDEGUIMARÃES; FARIA, 2007).

Alguns programas foram criados pelo Ministério da Saúde para melhorar a qualidade da triagem clínica e sorologia dos candidatos a doação de sangue. Inicialmente o Ministério da Saúde criou um Programa Nacional de Sangue e Hemoderivados e em 1980 houve a implantação da HEMORREDE, uma rede pública estadual hierarquizada de serviços de hemoterapia, atraindo assim recursos humanos qualificados, contribuindo para a redução da prevalência de positividade para $T$. cruzi entre os doadores (MORAES-SOUZA; FERREIRASILVA, 2011). Mesmo após a implantação destes programas, o risco de infecção pelo T. cruzi via transfusão de sangue ainda é de $12 \%$ a $25 \%$, sendo o desafio dos bancos de sangue a identificação e exclusão de portadores assintomáticos e crônicos do parasita (BLEJER; SAGUIER; SALAMONE, 2001). Atualmente, existem poucos estudos na literatura sobre a prevalência de doenças transmissíveis pelo sangue na população brasileira de doadores. $\mathrm{O}$ estudo da prevalência da infecção pelo T.cruzi em candidatos à doação de sangue tem se apresentado como um indicador sensível, podendo ser entendido como um verdadeiro marcador do risco da doença de Chagas transfusional e até mesmo como um medidor do nível de transmissão da doença em uma região (GUIMARÃES-SAÚDE; FARIA, 2007).

Quanto à prevenção da infecção congênita esta não possui medida preventiva, porém, entende-se que a detecção do caso e o tratamento específico de modo precoce seja o mais recomendado. Quando há suspeita clínica o ideal é realizar as provas sorológicas nas gestantes. Recém-nascidos de mães infectadas devem passar por exames parasitológicos logo ao nascer e, em casos positivos, iniciar o tratamento imediatamente. Em casos negativos para tripanossomíase a sorologia deve novamente ser feita aos sete ou oito meses de vida, quando anticorpos da classe de IgG não são mais considerados maternos e sim da própria criança sendo o tratamento instaurado imediatamente nos que forem positivos (DIAS; AMATO NETO; LUNA, 2011; GONTIJO et al, 2009).

Referente à prevenção da contaminação de $T$. cruzi por acidentes, pode-se verificar sua ocorrência de modo primário (antes que aconteça) e secundário (onde se previne o dano após o acontecimento do fato). No modo primário fatores como a adoção de medidas de biossegurança o uso de equipamentos de trabalho e de proteção individual o cumprimento de rotinas e o treinamento são indispensáveis tanto para técnicos, como para pesquisadores iniciantes. Uma vez cumpridos rigorosamente e em constante supervisão a ocorrência de acidentes primários cai consideravelmente. É importante ressaltar também a importância de teste sorológico do técnico iniciante, logo no começo do envolvimento com o parasito (DIAS; AMATO NETO, 2011). Já a prevenção secundária é de caráter prático, sendo adotadas uma série de providências quando surgem suspeitas de risco de contaminação. Por exemplo, em caso de ferimento ou contaminação externo ou ocular deve-se realizar a desinfecção local com álcool, mertiolate ou nitrato de prata, em seguida, fazer uma coleta para uma sorologia 
controle e posteriormente iniciar tratamento com o uso de benzonidazol ou nifurtimox em doses habituais por dez dias. Deve-se ressaltar que as autoridades sanitárias, bem como a comissão interna de biossegurança devem ser informados quando ocorrido a transmissão acidental para assim realizar investigação e avaliação do caso, transcendendo a correção dos fatores de risco (DIAS; AMATO NETO; LUNA, 2011).

\section{CONCLUSÃO}

Com base no que foi abordado, pode-se afirmar que a doença de Chagas seja pela prevalência, seja pela gravidade de suas manifestações, constitui, ainda hoje, um problema de saúde pública. Mesmo que a transmissão da doença de Chagas tenha declinado significativamente nos últimos trinta anos no Brasil e em grande parte da América Latina, a correta identificação dos portadores desta enfermidade e as ações de controle do triatomíneo são de fundamental importância no que tange à saúde pública em nosso país.

\section{REFERÊNCIAS BIBLIOGRÁFICAS}

AGÊNCIA NACIONAL DE VIGILÂNCIA SANITÁRIA. Ministério da Saúde. Resolução da Diretoria Colegiada n⿳0 57, 16 de dezembro de 2010. Disponível em:

<http://www.prosangue.sp.gov.br/uploads/legislacao>. Acesso em: 25 de set. 2012.

ALBAJAR, P. V.; LAREDO, S. V.; TERRAZAS M. B.; COURA, J. R. Miocardiopatia dilatada em pacientes com infecção chagásica crônica. Relato de dois casos fatais autóctones do Rio Negro, Estado do Amazonas. Revista da Sociedade Brasileira de Medicina Tropical. v.36, n⿳3 3, p. 401-407. 2003.

ALMEIDA B. R.; SANTILIANO F. C. Levantamento dos métodos de diagnóstico para a doença de Chagas. Enciclopédia biosfera, Centro Científico Conhecer - Goiânia. v.8, nº 14, p. 1586. 2012.

ARAS, R.; GOMES, I.; VEIGA, M.; MELO, A. Transmissão vetorial da doença de Chagas em Mulungu do Morro, Nordeste do Brasil. Rev. da Sociedade Brasileira de Medicina Tropical, v. 36, n 3, p. 359-363. 2003.

ARGOLO, A. M.; FELIX, M.; PACHECO, R.; COSTA, J. Doença de Chagas e seus principais vetores no Brasil. Rio de Janeiro. 2007. (Livro em processo de editoração e revisão).

ARAÚJO SM, GOMES ML, ORTIZ M, COSTA AL, MEMBRIVE UA, TOLEDO MJO, GUILHERME ALF. Doença de Chagas em crianças no Estado do Paraná, Brasil.

Revista da Sociedade Brasileira de Análises Clínicas 2004;36(3):151-153.

BARBOSA, L. G. N. DOENÇA DE CHAGAS. Revista Logos. nº.1, p. 20-36. 2009.

BARBOSA-FERREIRA, J. M.; GUERRA, J. A. O.; SANTANA FILHO, F. S.; MAGAlHÃES, B. M. L.; COELHO, L. I. A. R. C.; BARBOSA, M. G. Acometimento 
cardíaco em casos de doença de Chagas aguda da Amazônia. Arq. Bras. Card. v. 94, n 6, p. 147-149. 2010.

BEDIN, C.; WILHELMS, T. M. S.; TORRES, M. A.; ABBAD, P. R. S.; LIPPOLD, K. J.; SENFF, L. A.; SAKIS, P. R. A singularidade da melhoria habitacional para o controle da doença de Chagas na região noroeste do Rio Grande do Sul. Bol. da Saúde, v. 15, n 1 , p. 107-115. 2001.

BEZZERA, W. S.; MENEGUETTI, D. U. O.; CAMARGO, L. M. A. A busca de fármacos para tratamento da Tripanossomíase Americana: 103 anos de negligência. Revista Saúde (Sant Maria). v. 38, $\mathrm{n}^{\mathbf{o}}$ 1, p. 09-20. 2012.

BLEJER, SAGUIER, SALAMONE 2001. Antibodies to Trypanosoma cruzi among blood donors in Buenos Aires, Argentina. Int J InfectDis. v. 5, n 2, p. 89-93. 2001.

BRASIL, Fundação Nacional de Saúde. Manual de orientações técnicas para elaboração de projeto de melhoria habitacional para o controle da doença de Chagas. Brasília: Funasa, 2003. $53 \mathrm{p}$

BRASIL, MINISTÉRIO DA SAÚDE. Portal da Saúde. Aspectos epidemiológicos. Secretaria de Vigilância em Saúde. 2012. Disponível em:

<http://portal.saude.gov.br/portal/saude/profissional/visualizar_txto.cfm?idtxt=31454>.

Acesso em: 02 de out. 2012.

BRASIL. Consenso Brasileiro em Doença de Chagas. Secretaria de vigilância em saúde do ministério da saúde. Revista da Sociedade Brasileira de Medicina Tropical. v. 38, nº III, p.129. 2005.

BRASIL. MINISTÉRIO DA SAÚDE. Caderno de atenção básica: vigilância em saúde, zoonoses. Brasília. 2009.

BRASIL. MINISTÉRIO DA SAÚDE. Guia de vigilância epidemiológica. 7 ed. Brasília, DF. 2009. Disponível em:

<http://portal.saude.gov.br/portal/arquivo/pdf/gve_7ed_web_atual.pdf $>$. Acesso em: $08 \mathrm{de}$ out. 2012.

CAVALCANTE, M. P.; LORENA, V. M. B.; GOMES, Y. M. Avanços biotecnológicos para o diagnóstico das doenças infecciosas e parasitárias. Revista de Patologia Tropical. v. $37, \mathrm{n}^{\mathrm{o}} 1$, p. 1-14. 2008.

CAVALCANTI, L. P. G.; ROLIM, D. B.; PIRES NETO, R. J.; VILAR, D. C. L. F.; NOGUEIRA, J. O. L.; POMPEU, M. M. L.; TEIXEIRA, M. J.; SOUSA, A. Q. 
Microepidemia de doença de Chagas aguda por transmissão oral no Ceará. Cad. Saúde Coletiva, Rio de Janeiro, ${ }^{\circ}$ 17, v. 4, p. 911-921. 2009.

CIMERMAN, B.; CEMERMAN, S. Parasitologia Humana e seus Fundamentos Gerais. 2 ed. São Paulo: editora Atheneu, 2008. p. 81-112.

COLOSIO, R. C.; FALAVIGNA-GUILHERME, A. L.; GOMES, M. L.; MARQUES, D. S. O.; LALA, E. R. P.; ARAÚJO, S. M. Conhecimentos e atitudes sobre a doença de Chagas entre profissionais de saúde - Paraná, Brasil. Revista Ciência Cuidado e Saúde. v. 6, nº 2, p. $355-363.2007$.

DIAS, J. C. P. Doença de Chagas e transfusão de sangue no Brasil: vigilância e desafios. Revista Brasileira de Hematologia e Hemoterapia. v. 28, n 2. 2006

DIAS, J. C. P.; AMATO NETO, V. Prevenção referente às modalidades alternativas de transmissão do Tripanosoma cruzi no Brasil. Revista História sobre a Doença de Chagas no Brasil, v. 44, $\mathrm{n}^{\circ} 2.2011$.

DIAS, J. C. P.; AMATO NETO, V.; LUNA, E. J. A. Mecanismos alternativos de transmissão do Trypanosoma cruzi no Brasil e sugestões para sua prevenção. Revista da Sociedade Brasileira de Medicina Tropical, v. 44, n³ 3, p. 375-379, mai-jun. 2011.

FERREIRA, I.L.M.; SILVA, T.P.T. Eliminação da transmissão da doença de Chagas pelo Triatoma infestans no Brasil: um fato histórico. Revista da Sociedade Brasileira de Medicina Tropical. v. 39, n 5, p. 507-509. 2006.

FITARELLI, D. B.; HORN, J. F. Descarte de bolsas de sangue devido à reatividade para doença de Chagas em um laboratório de triagem sorológica de doadores em Porto Alegre-RS. Revista Brasileira de Hematologia e Hemoterapia. 2008.

GILBER, S. R. Reação em cadeia da polimerase em comparação com o teste de imunofluorescência indireta (IFI) e ELISA (enzimaimunoensaio) no diagnóstico para a doença de Chagas. Curitiba-PR.Dissertação em processos biotecnológicos, setor de tecnologia. Universidade Federal do Paraná. 2007.

GONTIJO, E. D.; ANDRADE, G. M. Q.; SANTOS, S. E.; GALVÃO, L. M. C.; MOREIRA, E. F.; PINTO, F. S.; DIAS, J. C. P.; JANUÁRIO, J. N. Triagem neonatal da infecção pelo Tripanosoma cruzi em Minas Gerais, Brasil: transmissão congênita e mapeamento das áreas endêmicas. Revista Epidemiol. Serv. Sáude, v. 18, nº 3, p. 243-245.2009. 
KROPF, S. P.; AZEVEDO, N.; FERREIRA L. O. Doença de Chagas: construção de um fato científico e de um problema de saúde pública no Brasil. Revista Ciência \& Saúde Coletiva. v. 5, $\mathrm{n}^{\circ}$ 2, p. 347-365. 2000.

LAMOUNIER, J. A.; MOULIN, Z. S.; XAVIER, C. C. Recomendações quanto à amamentação na vigência de infecção materna. Jornal de Pediatria, v. 80, nº 5, p. 181-188. 2004.

LOZANO, V. F. Avaliação da atividade antiparasitária e efeito sinérgico de compostos cumarínicos comparados ao benzonidazol em duas cepas de Trypanosoma cruzi. São Paulo. Dissertação. Universidade Bandeirantes de São Paulo. 2011.

LUNARDELLI, A.; BORGES, F. P.; MELLO, K. F.; ZEFERINO, A. S. A. Soroprevalência da doença de Chagas em candidatos a doadores de sangue. Revista Brasileira de Análise Clínica. v. 39, nº 2, p. 139-141. 2007.

MARKELL; VOGE; JOHN; KROTOSKI. Parasitologica médica. 8 ed. Rio de Janeiro: editora Guanabara Koogan. 2003. p.126-136.

MELO, A. S.; LORENA, V. M. B.; MORAES, A. B.; PINTO, M. B. A.; LEÃO, S. C.; SOARES, A. K. A.; GADELHA, M. F. S.; GOMES, Y. M. Prevalência de infecção chagásica em doadores de sangue no estado de Pernambuco, Brasil. Revista Brasileira de Hematologia e Hemoterapia. 2008.

MEYER, I. F.; KANESHIMA, E. N.; SOUZA-KANESHIMA, A. M. Alterações no sistema digestivo desencadeadas pelo quadro infeccioso do trypanosoma cruzi. Iniciação Científica Cesumar, v. 08, nº 01, p. 11-23, Jan-jun. 2006.

MORAES-SOUZA, H.; FERREIRA-SILVA, M. M. O controle da transmissão transfusional. Rev. História sobre a Doença de Chagas no Brasil, v. 44, supl. II, p. 64-67, 2011.

NASCENTE, F. M. Avaliação do perfil de parasitemia por hemocultura seriada em indivíduos infectados cronicamente pelo Trypanosoma cruzi. Goiânia-Go. Dissertação na área de concentração de parasitologia. Universidade Federal de Goiás. 2010.

NEVES, D. P.; MELO, A. L.; LINARDI, P. M.; VITOR, R. W. A. Parasitologia Humana. 11 ed. São Paulo: editora Atheneu. 2005. p. 85-108.

OLIVEIRA, M. F.; NAGÃO-DIAS, A. T.; PONTES, V. M. O.; SOUZA, J. A. S.; COELHO, H. L. L.; COELHO, I. C. B. Tratamento etiológico da doença de Chagas no Brasil. Revista de Patologia Tropical. v. 37, nº 3, p. 2009-228. 2008. 
OPAS - Organización Panamericana de laSalud. Consulta Técnica sobre Información, Educación y Comunicación (IEC), em Enfermedad de Chagas Congénita. Montevideo, Uruguay; 2007.

ORGANIZAÇÃO PAN-AMERICA DA SAÚDE. Doença de Chagas: guia pra vigilância, prevenção, controle e manejo da doença de Chagas aguda transmitida por alimentos. Rio de Janeiro: PANAFTOSA-VP/OPAS/OMS, 2009.

PINTO, A. Y. N.; VALENTE, S. A.; VALENTE, V. C.; FERREIRA JUNIOR, A. G.; COURA, J. R. Fase aguda da doença de Chagas na Amazônia brasileira. Estudo de 233 casos do Pará, Amapá e Maranhão observados entre 1988 e 1995. Revista da Sociedade Brasileira de Medicina Tropical. v. 41, n 6, p. 602-614. 2008.

PONTES V. M. O.; SOUZA J. A. S. CRUZ F. M. T.; COELHO L. L.; DIAS A. T. N.; COÊLHOS I. C. B.; OLIVEIRA M. F. Reações adversas em pacientes com doença de Chagas tratados com benzonidazol, no Estado do Ceará. Revista Brasileira de Medicina Tropical. v. 43, nº 2, p. 182-187. 2010.

REY, L. Parasitologia: parasitos e doenças parasitárias do homem nos trópicos ocidentais. 4 ed. Rio de Janeiro: editora Guanabara koogan. 2008. p. 295-343.

SANCHEZ-LERMEN R. L. P.; DICK E.; SALAS J. A. P.; FONTES C. J. F. Sintomas do trato digestivo superior e distúrbios motores do esôfago em pacientes portadores da forma indeterminada da doença de Chagas crônica. Revista da Sociedade Brasileira de Medicina Tropical. v. 40, nº 2, p. 197-203. 2007.

SANTOS, C. A. T.; Infecção pelo Trypanosoma cruzi em doadores de sangue: revisão sistemática da literatura e estudo de prevalência no hemocentro de Goiás. Goiânia-Go. Dissertação de mestrado em ciência da saúde. Universidade Federal de Goiás. 2011.

SAÚDE-GUIMARÃES, D. A.; FARIA, A. R. Substâncias da natureza com atividade antiTryppanosoma cruzi. Revista Brasileira de Farmacognosia, v. 17, n 3, p. 455-465, jul-set. 2007.

SILVA P. Farmacologia. 8 ed. Rio de Janeiro: editora Guanabara Koogan. 2010. p. 11071108.

SILVA, E. M.; ROCHA, M. O. C.; SILVA, R. C.; PAIXÃO, G. C.; BUZZATI, H.; SANTOS, A. N.; NUNES, M. C. P. Estudo clínico-epidemiológico da doença de Chagas no distrito de Serra Azul, Mateus Leme, centro-oeste do estado de Minas Gerais. Revista da Sociedade Brasileira de Medicina Tropical. v. 43, n² 2, p. 178-181. 2010. 
SILVA, M. M. F. Análise do desempenho de testes imunodiagnosticos para doença de Chagas e sua correlação com dados epidemiológicos para definição do perfil sorológico de doadores de sangue. Tese em Patologia Clínica. Universidade Federal do Triângulo Mineiro. Uberaba-MG. 2011.

SILVA, V. L. C. Subsídios ao processo de certificação do Brasil como área livre da transmissão transfusional da doença de Chagas. 2010. Dissertação (Mestrado em Saúde Coletiva) - Faculdade de Ciências Médias da Santa Casa de São Paulo. São Paulo. 2010.

SILVEIRA, A. C. Os novos desafios e perspectivas futuras do controle. Rev. História sobre a Doença de Chagas no Brasil, v. 44, supl. II, p. 122-124, 2011.

SILVEIRA, A. C.; DIAS, J. C. P. O controle da transmissão vetorial. Rev. História sobre a Doença de Chagas no Brasil, v. 44, supl. II, p. 52-63, 2011.

SOBREIRA, A. C. M.; GOMES, V. B. A. F.; SILVA, M. A. M.; OLIVEIRA, M. F. Prevalência de infecção chagásica em doadores de sangue do Hemocentro Regional de Iguatu, CE. Revista da Sociedade Brasileira de Medicina Tropical, v. 34, n 2, p. 193-196, mar-abr. 2001.

SOUSSUMI, L. M. T. Estudo da distribuição de doadores reativos para a doença de Chagas no hemocentro de Ribeirão Preto-SP. Ribeirão Preto-SP. Dissertação em Saúde da Comunidade, departamento de Medicina Social. Faculdade de Medicina de Ribeirão Preto, Universidade de São Paulo. 2004.

TARTAROTTI, E.; AZEREDO-OLIVEIRA, M. T. V., CERON, C. R. Problemática vetorial da doença de Chagas. Arq. Ciênc. Saúde, v. 11, nº 1, p. 44-47. 2004. 